\title{
Multi-Component V2X Applications Placement in Edge Computing Environment
}

\author{
Ibrahim Shaer*, Anwar Haque ${ }^{\dagger}$, and Abdallah Shami* \\ ${ }^{*}$ Department of Computer and Electrical Engineering, ${ }^{\dagger}$ Department of Computer Science \\ Western University, London ON, Canada \\ \{ishaer, ahaque32, abdallah.shami\}@uwo.ca
}

\begin{abstract}
Vehicle-to-everything (V2X) services are attracting a lot of attention in the research and industry communities due to their applicability in the landscape of connected and autonomous vehicles. Such applications have stringent performance requirements in terms of complex data processing and low latency communications which are utilized to ensure road safety and improve road conditions. To address these challenges, the placement of V2X applications through leveraging of edge computing paradigm, that distributes the computing capabilities to access points in proximity to the vehicles, presents itself as a viable solution. However, the realistic implementation of the edge enabled V2X applications is hindered by the limited computational power provided at the edge and the nature of V2X applications that are composed of multiple independent V2X basic services. To address these challenges, this work targets the efficient placement of $\mathrm{V} 2 \mathrm{X}$ basic services in a highway scenario subject to the delay constraints of $\mathrm{V} 2 \mathrm{X}$ applications using them and the limited computational resources at the edge. To that end, this work formulates a binary integer linear programming model that minimizes the delay of $\mathrm{V} 2 \mathrm{X}$ applications while satisfying the resource requirements of $\mathrm{V} 2 \mathrm{X}$ basic services. To demonstrate the soundness of the approach, simulations with varying vehicle densities were conducted, and the results reported show that it can satisfy the delay requirements of $\mathrm{V} 2 \mathrm{X}$ applications.
\end{abstract}

Index Terms-V2X applications, edge computing, placement, multi-component

\section{INTRODUCTION}

Intelligent Transportation Systems (ITSs) are envisioned to ameliorate traffic congestion and improve road safety and traffic experience. ITSs have drawn the attention of a large number of stakeholders due to their direct effect on the manufacturing of sensor and wireless-equipped vehicles known as connected and autonomous cars. In this regard, Vehicle-toeverything (V2X) applications are considered a key enabler for the shift to Intelligent Transportation Systems (ITSs) in terms of traffic management. These applications allow the vehicles to communicate and exchange information with their surrounding environment that includes other vehicles, pedestrians and supporting road side units (RSUs). To ensure road safety, these applications operate with stringent end-to-end (E2E) latency/delay. There are different paradigms that can determine the placement of V2X applications to address the E2E latency requirements. The placement of these services is disruptive to the customary cloud-based infrastructure. The projected increase in the number of connected and autonomous vehicles will result in data explosion. The data will be routed to a single centralized server creating severe network traffic congestion
[1] . Additionally, the centralized servers are usually located far from vehicles generating data; thus, incurring a huge E2E delay. Furthermore, this architecture exposes a single point of failure, which is huge risk to take for time and mission-critical V2X applications. Given these circumstances, distributing the cloud computing technology in proximity to users is proposed as a viable solution to deal with the shortcomings of the centralized paradigm [2]. This computing architecture is referred to as Edge Computing. Edge Computing can support the latency requirements of the V2X applications which are critical for their performance [3]. In addition, the edge servers collect data from the close local nodes which allows for a more individualized experience for the V2X application users. While Edge Computing paradigm can ensure some V2X system-level performances, this comes at the expense of limited computational power at the edges which hinders the processing of large amount of data. Microservices architecture, that decomposes a single application into decoupled modules, combined with virtualization techniques, that fully utilizes resource at the edges, can be used to address this issue. Hawilo et. al [4] investigated the applicability of this paradigm for Virtual Network Functions which display similar characteristics to $\mathrm{V} 2 \mathrm{X}$ applications making it a viable option for their placement. In the domain of V2X applications, 3rd Generation Partnership Project (3GPP) [5] envisions complex V2X applications that combine vehicle status analysis, imminent traffic events generation, and raw sensor data exchange that define the function of autonomous and connected vehicles. Each of these applications rely on the data processing and analysis of miniscule V2X basic services.

Mobile edge clouds (MEC), edge clouds and roadside cloud have been proposed in several previous works in the context of vehicular applications. In [6], Emara et. al employ an MEC-assisted architecture to evaluate end-to-end latency for vehicles to detect the vulnerable road units. Moubayed et. al [7] formulated an integer linear programming problem for efficient placement of $\mathrm{V} 2 \mathrm{X}$ basic service taking into consideration V2X basic services' delay and computational requirements in a hybrid environment that includes edge and core nodes. Supporting V2X applications while considering the vehicle's mobility aspects has been extensively addressed in literature. To support V2X applications, [8]-[10] consider migrating the services according to vehicle's mobility. In [8], the authors customize a three-layered architecture that consists 
of a vehicular cloud, a roadside cloud and a central cloud to support vehicular applications. Their approach focuses on the dynamic allocation of resources, driven by the vehicle's mobility, in vehicular and roadside clouds. In [9], Yu et. al consider the migration of $\mathrm{V} 2 \mathrm{X}$ applications placed on edge servers according to predictive vehicle's mobility combined with setting a priority schema for V2X applications. The approach considers the latency and resource requirements of each of the applications. In the same context, Yao et. al [10] investigate Virtual Machine (VM) placement and migration in roadside cloud that is part of the vehicular cloud computing architecture. The approach targets minimizing the overall network cost given the available resources at the edge. Each of these previous works has its own shortcoming. One common aspect is considering either latency or resource limitations for the placement of the services at the edge. Another shortcoming is the disregard of the nature of $\mathrm{V} 2 \mathrm{X}$ applications that may be composed of a single or many modules. Finally, different traffic conditions were not considered to model any solution for vehicular application placement. To address these shortcomings, this work focuses on V2X application placement that minimizes the end-to-end delay which takes into consideration the computational requirements of $\mathrm{V} 2 \mathrm{X}$ services forming it. This work's main contributions are as follows:

- Decompose V2X applications into multi-V2X basic services.

- Formulate the optimal V2X application placement by considering their delay requirements and the resource requirements of their constituent components.

- Evaluate the performance of the optimal placement in terms of average delay and density distribution for each $\mathrm{V} 2 \mathrm{X}$ application under different traffic conditions.

The remainder of this paper is organized as follows: Section II describes the system model and presents the problem formulation, Section III provides the simulation procedure and discusses the results and Section IV concludes the paper and suggests future work.

\section{SySTEM MODEL}

In the reference model, a highway scenario is considered. Each of the vehicles moving on the highway is running a set of V2X applications that are collecting data from nearby roadside units (RSUs) to function autonomously. RSUs and the vehicles are communicating directly using Dedicated Short-range Communication [11], and no communication takes place between any vehicles. Each RSU is equipped with a server which are both considered as an edge computing node. Vehicles are receiving data from $\mathrm{V} 2 \mathrm{X}$ basic services placed on each RSU. European Telecommunication Standardization Institute (ETSI) defines three V2X basic services that are the foundation of any envisioned V2X applications. The V2X basic services are as follows: Cooperative Awareness Basic Service (CA) [12] is responsible for creating, analyzing and sending Cooperative Awareness Basic Messages (CAMs) which include information about the vehicle's status and attributes, Decentralized Environmental Notification Service [13] (DEN) broadcasts
Decentralized Notification Messages (DENM) whenever a road hazard or abnormal traffic condition takes place, and Media Downloading [14] service is requested on demand by the passengers of the vehicle. Additionally, ETSI defines Local Dynamic Maps (LDMs) [15] that are responsible for storing the sent CAMs and DENMs. Because LDMs store spatial relevant information, an LDM is deployed on each edge server. LDMs are queried by V2X basic services in order to retrieve information. Finally, in addition to basic vehicular services that are related to road safety, there is a variety of innovative applications that are referred to as value-added services that are of lower priority [16]. These services include augmented reality, parking location and others that are part of the infotainment services provided by vehicular applications. Compared to road safety applications, these services display high levels of diversity and individuation. Therefore, they need to be migrated when the vehicle moves from one edge server coverage zone to another. For this purpose, each edge server reserves part of its resources to accommodate these migrating services. In this section, the system design and the optimal optimization technique for V2X basic service placement are presented.

\section{A. System Design}

In the reference model used for the placement of $\mathrm{V} 2 \mathrm{X}$ basic services, HWY 416 IC-721A that passes through the city of Ottawa is considered. The edge computing servers are deployed uniformly along the highway as the deployment of RSU is out of the scope of this paper. No communication interference zone exists between any two successive RSUs to avoid the possibility of encountering ping-pong handover cases which will be difficult to handle in an optimization model. Additionally, the vehicles are assumed to be always connected to RSUs throughout their journey. The end-to-end latency of a service is the sum of the communication, processing, transmission and propagation delay. The propagation delay is dependent on the medium of communication which is out of the scope of this paper, and therefore considered negligible. In this model, DSRC, the communication technology between the moving vehicles and RSUs, affects the communication delay. In the proposed model, the processing and transmission delay between the communicated edge servers is considered. Each edge computing server has the same computing and processing power that are expressed by the number of cores and RAM available. Finally, the vehicle density is considered to model real case scenarios.

\section{B. Optimization Problem}

In the optimization function, a set of edge servers and V2X services are considered. Let $N$ denote the set of edge servers where $n \in N$. Let $U$ denote the set of unique V2X basic services where $u \in U$. The availability of the computational resources on the edge is denoted by matrix Cap where $C a p_{k n}$ denotes the $k$ th computational resources available on edge server $n$. Matrix $R$ represents the resources required by the V2X basic services where $R_{k u}$ represents the 
$k$ th computational resources required by V2X basic service $u$. A binary row vector $\vec{q}$ denotes the edge servers a vehicle can communicate with. Let $C$ be the matrix that represents the processing and the transmission latency between edge servers where $C_{i j}$ represents the latency between edge server $i$ and edge server $j$. Matrix $M$ represents the V2X services needed by V2X applications where $M_{a u}=1$ denotes that application $a$ needs V2X basic service $u$. Let $X$ denote the placement matrix where $X_{u n}=1$ means that V2X basic service $u$ is placed on edge server $n$. The column $X_{u}$ denotes the placement of the V2X services on edge server $n . D_{a}^{v}$ and $D_{a}^{t h}$ denote respectively the delay experienced by a moving vehicle $v$ served by application $a$ and the maximum tolerable threshold of this delay. To represent the vehicles' density, $\gamma$ is used. $d_{c o m}^{v}$ and $d_{D L}^{v}$ denote respectively the communication and download latency between a vehicle $v$ and a serving edge server. The optimization function used to minimize the delay of V2X application is as follows:

$$
\min \sum_{a \in A} D_{a}^{v}
$$

where:

$D_{a}^{v}=d_{c o m}^{v}+\max \left(M_{a} \odot \min (X \odot(\gamma C \times \vec{q}))+d_{D L}^{v}\right.$

subject to:

$$
\begin{gathered}
D_{a}^{v} \leq D_{a}^{t h}, \forall a \in A \\
R X \leq C a p \\
\sum X_{n}=1, \forall n \in N
\end{gathered}
$$

In what follows, the explanation of the equations (1)(5). Equation (1) describes the overall objective which is minimizing the summation of the delay of all V2X application experienced by a vehicle requesting their services. Equation (2) presents the components contributing to the delay of V2X application. The delay of a V2X application is the delay of the V2X services it relies on depending on the edge servers a vehicle can communicate with. Because the functioning of a V2X basic service is independent of other V2X basic services, the delay of a V2X application is defined as the maximum of the delay of its constituent V2X basic services. This value is added to the communication and download link delay. Next, the equations (3) - (5) describe the constraints. Equation (3) defines that the delay of an application should not exceed its maximum defined tolerable delay. Equation (4) ensures that the resources allocated to a V2X service do not exceed the available resource on the hosting edge server. Equation (5) limits placing only one V2X service on each edge server. The following diagram illustrates an example of the communication and processing that takes place for a V2X application that requires CA and DEN services, given that each server has resources reserved for migrating applications
Fig. 1. System Model

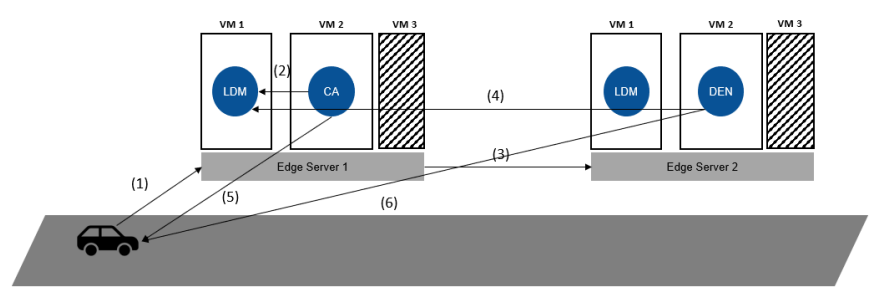

denoted by VM 3. The logic governing the realization of the application is as follows:

(1) The vehicle requests the services of an application. This step incurs communication delay that is denoted by $d_{c o m}^{v}$.

(2) The CA service found on Edge Server 1 requests the necessary information from the LDM. The processing of the request on the $\mathrm{LDM}$ is denoted by $C_{11}$.

(3) Edge server 1 communicates with the closest server that include DEN basic service. No delay is considered in this phase.

(4) DEN queries and receives information from the LDM that is closest to the requesting vehicle. The LDM on edge server 1 has accurate information about the requesting vehicle's surrounding environment. This delay is the sum of the processing delay of LDM on edge server 1 and the transmission delay between edge server 1 and 2 . This is denoted by $C_{12}$.

$(5,6)$ These steps represent the CA and DEN response to the requesting vehicle. This delay is denoted by $d_{D L}^{v}$.

For basic service CA, the delay is as follows:

$$
d_{C A}^{v}=C_{11}+d_{D L}^{v}
$$

Similarly, the delay for DEN is:

$$
d_{D E N}^{v}=C_{12}+d_{D L}^{v}
$$

Given that the requests for each basic service are executed in parallel and that these services are independent in their execution, the delay experienced by a vehicle $v$ requesting the services of application $a$ is:

$$
D_{a}^{v}=d_{c o m}^{v}+\max \left\{d_{C A}^{v}, d_{D E N}^{v}\right\}
$$

\section{EXPERIMENTAL SETUP AND RESUlts}

\section{A. Simulation Setup}

In order to evaluate the placement of V2X basic services, a realistic simulation environment must be created. To this end, Simulation of Urban Mobility (SUMO) [17] was used to extract the movement of vehicles along a highway. A 4 km highway that resembles HWY 416 IC-712A was considered as a reference highway. Ontario traffic volume for provincial highways [18] provided the average daily traffic and the accident rates during summer, winter, weekdays and weekends. In the simulation setup, the statistics offered by 
TABLE I

SUMO VEHICLE MOVEMENT PARAMETERS

\begin{tabular}{|c|c|}
\hline Parameter & Value \\
\hline \hline Maximum Speed & $27.7 \mathrm{~m} / \mathrm{s}$ \\
\hline Maximum Acceleration & $2.6 \mathrm{~m} / \mathrm{s}^{2}$ \\
\hline Maximum Deceleration & $4.5 \mathrm{~m} / \mathrm{s}^{2}$ \\
\hline
\end{tabular}

this report were used to emulate moderate and heavy traffic experienced on HWY 416 IC-712A highway that is expressed through the vehicles per hour parameter in SUMO. Regarding the movement of the vehicles, Table I summarizes the key parameters that are used in the simulation.

The V2X applications considered are Platooning (PL), Sensor and Sensor State Mapping (SSM), Emergency Stop (ES), Pre-crash Sensing Warning (PSW) and Forward Collision Warning $(\mathrm{FCW})$. Their corresponding performance requirements and service components are presented in Table II [19], [20]. Choosing these V2X applications stems from their importance and stringent performance requirements in the realm of the autonomous cars. In addition, in the context of the defined problem, each of the chosen V2X application offers a unique combination of V2X services. In the simulation procedure, the communication delay between a vehicle and an RSU is $1 \mathrm{~ms}$ [21]. In this model, the processing delay is the amount of time required by a Local Dynamic Map to process the data requested by other $\mathrm{V} 2 \mathrm{X}$ services either placed on the same or different edge server. In [22], the authors devise an LDM according to the specifications defined by ETSI. The application defines two Application Programming Interfaces (APIs) that retrieve information of the IDs of the vehicles driving on the same road and the vehicle driving immediately ahead of the requesting vehicle. For different number of queried vehicles ranging from 5 to 20 vehicles, the response time was between 3 and $5 \mathrm{~ms}$ with no clear correlation between the size of the data and the response time. Consequently, in the simulation setup, the processing delay is generated uniformly between 3 and $5 \mathrm{~ms}$. In the same context, the authors in [23], assumed the transmission latency between two edge servers to be between 1 and $5 \mathrm{~ms}$. Because the simulation procedure takes place under several vehicle densities, the increase of the data processing and transmission overhead with the increase of number of vehicles is inevitable. In this regard, the execution cost increases with the number of vehicles in proximity to the vehicle requesting the $\mathrm{V} 2 \mathrm{X}$ application services. As the implementation of LDM did not consider cases beyond 20 vehicles, the added delay for these cases will be in the form of $\log (N C / 20)$ where $N C$ represents the number of cars and the expression is derived from the increase of processing delay upon the increase in size of the queried data in SQL [24]. In terms of edge servers, 10 edge servers are deployed every 400 $m$ alongside the highway. Each of the RSUs hosts an LDM, V2X service and an optional migrating service. The computational requirements of CA, DEN and Media services are those of a small, medium and large VMs. Table III summarizes the edge server capabilities and the computational requirements of
TABLE II

BREAKDOWN OF V2X APPLICATIONS' V2X BASIC SERVICE AND PERFORMANCE METRICS

\begin{tabular}{|c|c|c|c|}
\hline Application & Service(s) & Latency(ms) & Reliability(\%) \\
\hline \hline PL & CA & 50 & 90 \\
\hline SSM & CA, DEN, Media & 20 & 90 \\
\hline ES & DEN & 10 & 95 \\
\hline PSW & CA, DEN & 20 & 95 \\
\hline FCW & CA, DEN & 10 & 95 \\
\hline
\end{tabular}

TABLE III

COMPUTATIONAL REQUIREMENTS

\begin{tabular}{|c|c|c|}
\hline Entity & Number of Cores & RAM \\
\hline \hline Edge Server & 8 & 8 \\
\hline CA & 2 & 2 \\
\hline DEN & 2 & 4 \\
\hline Media Service & 4 & 6 \\
\hline LDM & 4 & 2 \\
\hline
\end{tabular}

CA, DEN and Media services. In the experimental procedure, the placement of the V2X basic services is carried out using the defined optimization function. Next, traffic simulation is executed for defined densities that reflect moderate and heavy traffic. The traffic traces were generated for 1500 seconds. Every 10 seconds, a snapshot of the road condition is taken and delays for each V2X application for each vehicle is calculated. Finally, at the end of the simulation, the average delay for each $\mathrm{V} 2 \mathrm{X}$ application is obtained.

\section{B. Implementation}

The optimization function was solved using IBM ILOG CPLEX 12.9.0 through its Python API. The solution is provided instantly for all simulation scenarios with different vehicle densities on a laptop with an Intel Core i7-8750 CPU, $2.21 \mathrm{GHz}$ clock frequency and $16 \mathrm{~GB}$ of RAM. The final solution includes the V2X services placed on each edge server.

\section{Results and Discussion}

To evaluate the efficacy of the optimization function, the simulation procedure was carried out using two different traffic scenarios each representing moderate (Scenario 1) and heavy (Scenario 2) traffic models. The results are obtained as an average for five independent runs. To assess the placement function, the average delay of each V2X application under study is obtained and compared it to the maximum tolerable delay. Additionally, the model is evaluated using the probability density function of each of V2X application. The density function provides a more thorough overview about the distribution of the delays in terms of detecting extreme values that are overshadowed by the common values trend. Furthermore, the density functions reveal the shortcomings of the approaches that are concealed by the calculation of the mean. The suggested optimization function failed to converge, so a new heuristic algorithm that relaxes the delay threshold for each application by magnitudes of the reliability metrics is considered and executed. This heuristic algorithm is referred to as: Resource and Delay-aware V2X basic service Placement 
Fig. 2. Average Delay of V2X Applications for different Vehicle Densities

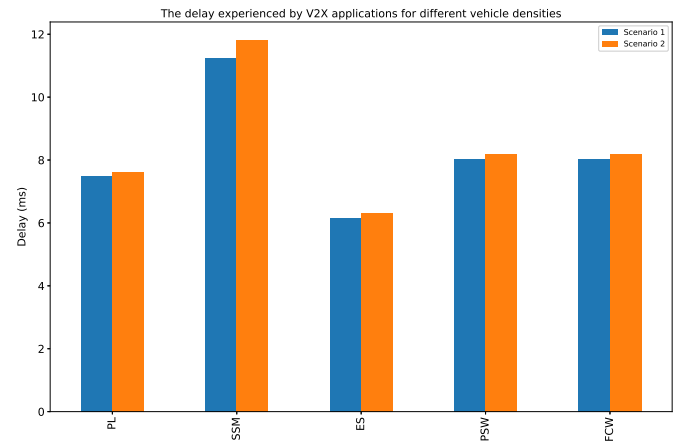

Fig. 3. Probability Density Function for 1500 vehicles/hour
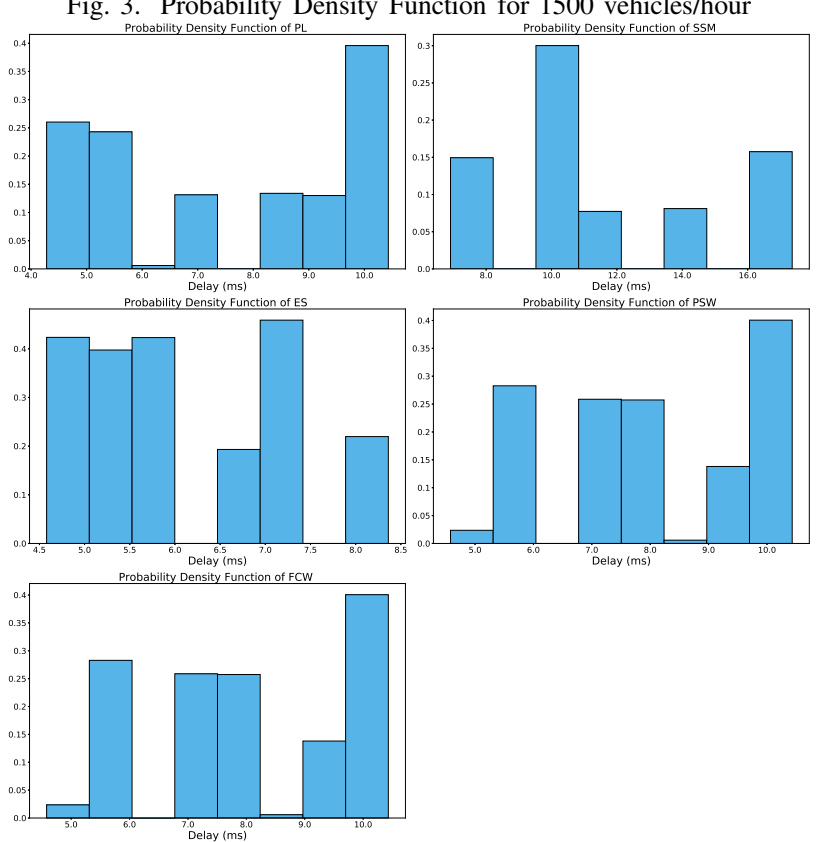

(RDP). The results of the simulation process in terms of the average delay and the probability densities of each V2X application are presented in Figures 2-5.

Figure 2 shows the mean delay for each of the V2X applications. The results clearly show that the average delay experienced by each V2X application is within the tolerable threshold. These results show that the heuristic algorithm met the stringent V2X application delay requirements. In terms of the traffic effect, the mean of delay of each application has slightly increased but still fulfills the overall objective of the placement function. The vehicles' density has contributed to an increase in the average delay of the V2X applications in the range of $1.3 \%$ to $4.8 \%$ whereby the SSM application has experienced the greatest variation. This fact shows that the placement of Media Service, that SSM relies on, is the most sensitive to traffic variation. On the other hand, the probability density functions tell a different story. Figures 3 and 4 depicting the delay distribution for both cases show that the delay is highly skewed to the left which supports the viability of the approach. However, this is not the case
Fig. 4. Probability Density Function for 1800 vehicles/hour
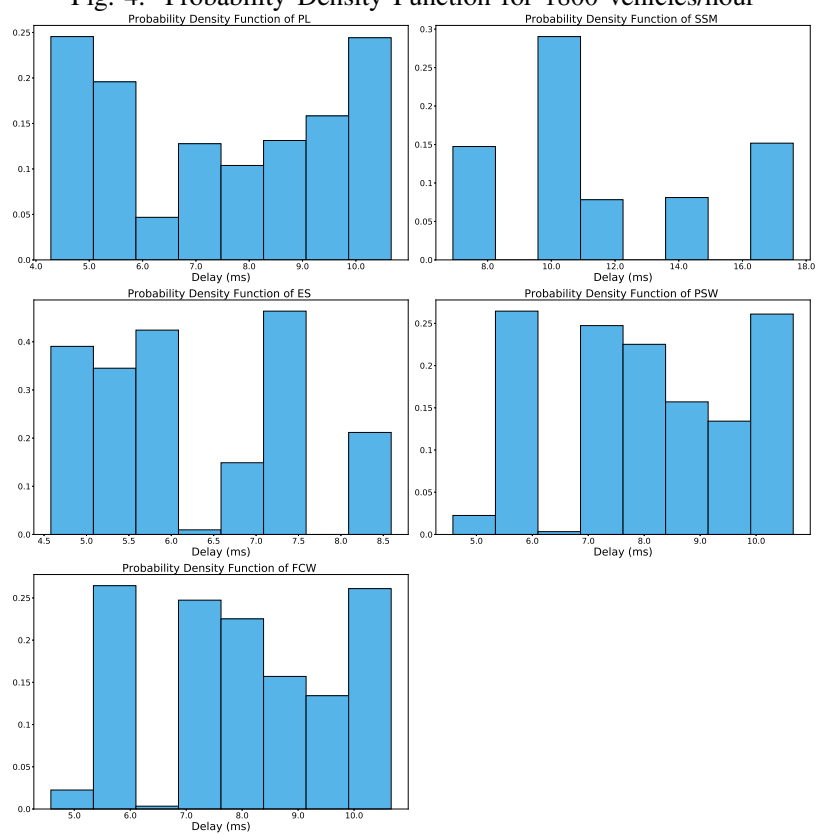

for FCW application which shows that for each scenario, $20 \%$ and $25 \%$ of the experienced delay exceeds the tolerable threshold which is beyond the 5\% permitted shown in Table II. In terms of traffic effect, it is observed that there is a slight right shift of the probability distribution in scenario 2 . Additionally, it is observed that some applications have similar probability distributions. This is attributed to the fact that these applications need the same V2X services, and as it shows, these services incur the most delay out of the other services that they rely on. The dispersion of some of the probability density function is due to the limited number of edge nodes hosting V2X services. The limited number of edge servers means that vehicles at the start and the end of the route will suffer from prolonged delay due to the distance separating the vehicles and the closest V2X basic services. In the cases of continued route, the suggested approach can be replicated along the highway to ensure that V2X services are delivered as expected. For comparison purposes and to further cement this paper's approach, a baseline approach that maximizes the resource utilization at each node server is compared to RDP. The baseline approach formulates a placement algorithm that takes into consideration only the available resources at each node. This baseline approach is reffered to as ResourceAware Algorithm (RAA). The two approaches were evaluated according to the probability density functions of the delays of ES and FCW applications. The probability densities are depicted in Figure 5.

The baseline line approach's density function shows promising results regarding the ES application as the full delay distribution is below the tolerable threshold. More values are concentrated on the extremes which makes it harder to gauge its value whenever the application is requested. However, for the case of FCW, this approach fails to be within the tolerable 
Fig. 5. RDP vs RAA
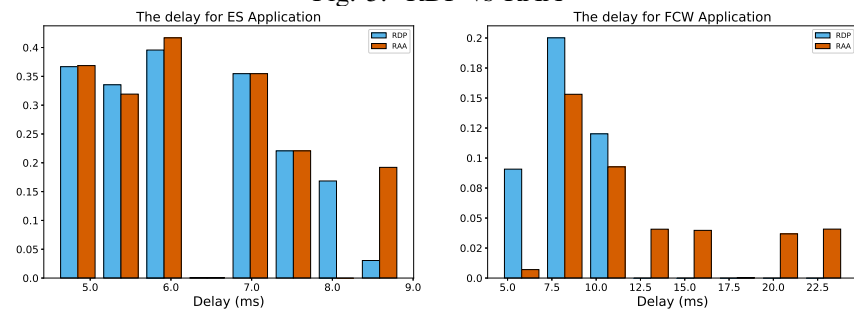

threshold rendering this approach ineffective for missioncritical applications. This is to be expected given that FCW application requires CA and DEN basic services. Due to the nature of RAA that maximizes the overall resource utilization, deploying more of CA services results in decreasing the utilization which incurs extra delay for FCW application when requesting the services of $\mathrm{CA}$.

\section{CONCLUSION}

This paper addressed the efficient placement of V2X basic service comprising different V2X applicatins in an edge computing environment. To this end, an optimization function that minimizes the delay for multi-component V2X applications consisting of $\mathrm{V} 2 \mathrm{X}$ services while considering the resource requirements of these services under different traffic conditions is formulated. The approach was evaluated under realistic scenarios where homogeneous edge servers with limited computational power and variable traffic conditions were considered. Furthermore, the approach was compared to a baseline approach that maximizes the overall resource utilization of edge servers. The results have shown that the approach guarantees an acceptable quality of service, and outperforms other approaches while emulating realistic conditions. While the current work considers that each V2X application has a constant request rate, the plan is to extend the work to consider different request distributions to mimic a real-world scenario. In the same context, the deployment of V2X applications in a dynamic service availability environment is also a subject to our future work.

\section{REFERENCES}

[1] Barrachina, J., Garrido, P., Fogue, M., Martinez, F., Cano, J., Calafate, C., and Manzoni, P, "Road Side Unit Deployment: A Density-Based Approach". IEEE Intell. Transp. Syst. Mag. 2013, 5, 30-39.

[2] Faticanti F., De Pellegrini F., Siracusa D., Santoro D. , and Cretti S., "Cutting throughput on the edge: App-aware placement in fog computing". CoRR, abs/1810.04442, 2018.

[3] Sabella D., Moustafa H., Kuure P., Zhou Z., Li A. et al. "Toward fully connected vehicles: Edge computing for advanced automotive communications ," 5GAA, December 2017. [Online]. Available: https://5gaa.org/wp-content/uploads/2017/12/5GAA_T-170219whitepaper-EdgeComputing 5GAA.pdf.

[4] Hassan Hawilo, M. Jammal and A. Shami, "Exploring Microservices as the Architecture of Choice for Network Function Virtualization Platforms," in IEEE Network, vol. 33, no. 2, pp. 202-210, March/April 2019.

[5] Study LTE Support for Vehicle to Everything (V2X) Services (Release 14), document 3GPP TR 22.885 V14.0.0, Dec. 2015.

[6] M. Emara, M. C. Filippou, and D. Sabella, "MEC-Assisted End-to-End Latency Evaluations for C-V2X Communications," in 2018 European Conference on Networks and Communications (EuCNC), Jun. 2018, pp. $1-9$.
[7] A. Moubayed, A. Shami, P. Heidari, A. Larabi, and R. Brunner, "Edgeenabled V2X Service Placement for Intelligent Transportation Systems." in IEEE Transactions on Mobile Computing.

[8] R. Yu, Y. Zhang, S. Gjessing, W. Xia, and K. Yang, "Toward cloudbased vehicular networks with efficient resource management," IEEE Netw., vol. 27, no. 5, pp. 48-55, Sep./Oct. 2013.

[9] X. Yu, M. Guan, M. Liao, and X. Fan, "Pre-migration of vehicle to network services based on priority in mobile edge computing," IEEE Access, vol. 7, pp. 3722-3730, 2019.

[10] H. Yao, C. Bai, D. Zeng, Q. Liang, and Y. Fan, "Migrate or not? exploring virtual machine migration in roadside cloudlet-based vehicular cloud," Concurrency and Computation: Practice and Experience, 27(18):57805792, 2015

[11] Z. Xu, X. Li, X. Zhao, M. H. Zhang, and Z. Wang, "DSRC versus 4G-LTE for Connected Vehicle Applications: A Study on Field Experiments of Vehicular Communication Performance," Journal of Advanced Transportation, vol. 2017, pp. 1-10, 2017.

[12] ETSI EN 302 637-2 (V1.3.2), "Intelligent Transport Systems (ITS) Vehicular Communications: Basic Set of Applications - Part 2: Specification of Cooperative Awareness Basic Service," 650 Route des Lucioles F06921 Sophia Antipolis Cedex - FRANCE, Technical Report., 2014.

[13] ETSI EN 302 637-3 (V1.2.1), "Intelligent Transport Systems (ITS) Vehicular Communications: Basic Set of Applications - Part 3: Specifications of Decentralized Environmental Notification Basic Service," 650 Route des Lucioles F- 06921 Sophia Antipolis Cedex - FRANCE, Technical Report, 2014.

[14] ETSI TR 102638 (V1.1.1), "Intelligent Transport Systems (ITS) Vehicular Communications: Basic Set of Applications - Definitions," 650 Route des Lucioles F-06921 Sophia Antipolis Cedex - FRANCE, Tech. Rep., 2009.

[15] ETSI TR 102863 (V1.1.1), "Intelligent Transport Systems (ITS); Vehicular Communications; Basic Set of Applications; Local Dynamic Map (LDM); Rationale for and Guidance on Standardization”, 650 Route des Lucioles F- 06921 Sophia Antipolis Cedex - FRANCE, Technical report, 2011.

[16] L. Li, Y. Li, and R. Hou, "A Novel Mobile Edge Computing-Based Architecture for Future Cellular Vehicular Networks," 2017 IEEE Wireless Communications and Networking Conference (WCNC), 2017.

[17] D. Krajzewicz, J. Erdmann, M. Behrisch, and L. Bieker, "Recent development and applications of SUMO - Simulation of Urban MObility," International Journal On Advances in Systems and Measurements, vol. 5, no. 3\&4, pp. 128-138, December 2012.

[18] Ontario Ministry of Transportation, "Provincial Highways Traffic Volumes 1988-2016," December 31, 2016. [Online]. Available: http://www.raqsa.mto.gov.on.ca/techpubs/TrafficVolumes.nsf/fa02780864 7879788525708a004b5df8/88c66a2279555c798525788d0048cca4/\$FILE /Provincial\%20Highways \%20traffic\%20Volumes\%201988-2016.pdf.

[19] 3GPP, "Study Enhancement 3GPP Support for 5G V2X Services (Release 15)," TR 22.886 V15.1.0, March 2017.

[20] 3GPP, "Study Enhancement 3GPP Support for 5G V2X Services (Release 16)," TR 22.886 V15.1.0, December 2018.

[21] D. Martin-Sacristan, S. Roger, D. Garcia-Roger, J. F. Monserrat, A. Kousaridas, P. Spapis, S. Ayaz, and C. Zhou, "Evaluation of lte- advanced connectivity options for the provisioning of v2x services," in 2018 IEEE Wireless Communications and Networking Conference (WCNC), Apr. 2018, pp. 1-6.

[22] Shimada H. , Yamaguchi A., Takada H. , and Sato K., "Implementation and Evaluation of Local Dynamic Map in Safety Driving Systems," Journal of Transportation Technologies, vol. 05, no. 02, pp. 102-112, 2015.

[23] Maheshwari, S.; Raychaudhuri, D.; Seskar, I.; Bronzino, F., "Scalability and Performance Evaluation of Edge Cloud Systems for Latency Constrained Applications," In Proceedings of the 2018 IEEE/ACM Symposium on Edge Computing (SEC), Seattle, WA, USA, 25-27 October 2018; pp. 286-299

[24] "MySQL 8.0 Reference Manual::13.8.2 EXPLAIN Statement," MySQL. Accessed on: Oct. 19, 2019. [Online]. Available: https://dev.mysql.com/doc/refman/8.0/en/explain.html. 